\title{
Simulación en laparoscopía durante la formación del cirujano general. Revisión y experiencia inicial
}

Javier Chinelli*, Gustavo Rodríguez ${ }^{\dagger}$

\section{Resumen}

La simulación surge como una herramienta fundamental dentro de lo que implica el cambio de paradigma de enseñanza de la cirugía al que asistimos en la actualidad, basado en teorías educativas modernas, la seguridad del paciente y en el caso de la laparoscopía las dificultades propias de la técnica. El objetivo fundamental es trasladar el entrenamiento en habilidades motoras fuera del block quirúrgico, al laboratorio de simulación. Para ello, existen diversos modelos de simulación, entre los que destacamos los box trainers, que pese a su baja fidelidad, suman varias ventajas en relación con sus costos, disponibilidad y reutilización. En este artículo realizamos una revisión descriptiva del tema, incluyendo los diferentes modelos y métodos de entrenamiento, estrategias de evaluación, así como de nuestra experiencia inicial en la Clínica Quirúrgica 2 del Hospital Maciel.

Palabras clave: Entrenamiento simulado

Educación médica

Cirugía general

Competencia clínica

Laparoscopía

Key words: $\quad$ Simulation training

Education, medical

Clinical competence

General Surgery

Laparoscopy

* Asistente de Clínica Quirúrgica 2.

† Prof. Titular de Clínica Quirúrgica 2.

Clínica Quirúrgica 2. Hospital Maciel.

Los autores declaran no tener conflicto de interés.

Trabajo aprobado por el Comité de Ética del Hospital Maciel.

Correspondencia: Dr. Javier Chinelli. Correo electrónico: jchinelli01@hotmail.com

Recibido: $23 / 7 / 18$

Aprobado: 10/9/18 


\section{Introducción}

La formación integral del cirujano se sustenta en varios pilares, entre los que destacamos: la adquisición de conocimientos teóricos, la integración de valores y el desarrollo de habilidades tanto técnicas como no técnicas. En los últimos años es evidente la necesidad de un cambio en el paradigma de enseñanza de la cirugía con respecto al modelo de Halsted (1) ("ver un procedimiento, hacer uno y luego enseñarlo"), adoptado inicialmente por los programas de residencias médicas de Estados Unidos. La justificación de dicho cambio puede resumirse en tres puntos: a) La evolución de la teoría educativa: existen teorías modernas, validadas y aceptadas, respecto a la adquisición de habilidades motoras y destrezas quirúrgicas. Entre ellas, la de Fitts-Posner ${ }^{(2)}$, según la cual se transita por tres etapas: cognitiva, donde la atención se centra únicamente en la tarea a realizar, generalmente a través de movimientos erráticos; etapa de integración: en ella, el procedimiento se realiza en forma repetitiva ("deliberate practice") con menos errores e interrupciones; finalmente, la etapa de automatización: el individuo realiza una tarea con fluidez, casi sin enfocar su atención en "lo que debe hacer" puesto que gran parte de ella se centra en perfeccionar la técnica ya aprendida; b) Cambios socio-epidemiológicos y problemas éticos: todos ellos giran en torno a un factor común, la seguridad del paciente. Una investigación en Estados Unidos en $1999^{(3)}$ detectó que el número de muertes evitables (por errores médicos) excedía a la de otras causas relativamente frecuentes, como accidentes de tránsito y el cáncer de mama. Si a esto le agregamos el hecho de que los residentes de cirugía tienen un número limitado de horas de trabajo semanal por disposición legal, y que el desarrollo de la cirugía mínimamente invasiva implica que los mismos ahora deban aprender el doble de destrezas quirúrgicas en un período de tiempo relativamente breve, podremos entender que desde el punto de vista ético existan serios cuestionamientos a que los médicos realicen su "entrenamiento" o formación completamente en pacientes reales, tal como ha sucedido hasta ahora; c) Desafios de la cirugía laparoscópica ${ }^{(4)}$ : adquirir competencia en cirugía laparoscópica implica dominar ciertos aspectos que son inherentes a ella. El más notorio es la visión bidimensional de un campo operatorio que en realidad es tridimensional ${ }^{(5)}$, lo que dificulta sobre todo la percepción de profundidad. Se utilizan instrumentos largos, con mucho menor rango de movimientos de los miembros superiores, con notoria pérdida de la sensación táctil y el feedback, y sometidos al efecto "fulcrum", consistente en la inversión de los movimientos de la mano con respecto a los del extremo del instrumento utilizado. Sin duda alguna, estos tres elementos (teoría educativa, limitantes éticas, problemas de la cirugía laparoscópica) justifican ampliamente la necesidad de trasladar al menos una parte de la formación del cirujano fuera del block quirúrgico, en concreto a un laboratorio de simulación.

Una de las grandes ventajas del entrenamiento en laboratorio de simulación, por demás evidente en otros ámbitos como, por ejemplo, el de la aviación, es la de transformar el entorno en un lugar estructurado, seguro, en donde el individuo puede cometer errores que no resultan en un daño real al paciente, y controlado, dado que no se lo expondrá a imprevistos o situaciones críticas, permitiendo enfocar toda la atención en la adquisición de destrezas ${ }^{(6)}$. Otra ventaja consiste en reducir el tiempo en que se logra la curva de aprendizaje ${ }^{(7)}$, de capital importancia en aquellos procedimientos a los que el cirujano se enfrenta con poca frecuencia, optimizando además el tiempo de utilización del block quirúrgico.

La simulación consiste básicamente en presentar algo de forma tal que parezca real, o que se asemeje de la mejor forma posible a la realidad ${ }^{(8)}$. Para ello existen diversos modelos de simuladores, que básicamente pueden ser inanimados o vivos. Entre los primeros están los modelos de banca (como el "box trainer"), los cadavéricos y de realidad virtual. Los modelos vivos utilizan animales. Nos referiremos fundamentalmente a los "box trainers" por ser aquellos más ampliamente utilizados, como en nuestra institución.

\section{Modelos de banca ("box trainers") y programas de entrenamiento en habilidades básicas}

Este dispositivo, que consiste en una caja opaca e iluminada por dentro, simula una cavidad abdominal y presenta orificios prefabricados en su sector anterior a través de los que se colocan trócares de acceso. Incluye, también, una cámara y un monitor, además de utilizar instrumental quirúrgico real. Son especialmente útiles para entrenar destrezas básicas y suturas, así como algunos pasos específicos de procedimientos laparoscópicos complejos (por ejemplo, anastomosis digestivas y bilio-digestivas). Para ello se utilizan habitualmente materiales inertes, así como tejidos animales ex vivo. Como ventaja, además de la utilización de instrumental laparoscópico real, provee un buen feedback, sobre todo de las sensaciones denominadas "hápticas"(9) por el uso de dicho instrumental. Otras ventajas son el bajo costo de adquisición y mantenimiento, su fácil traslado y la posibilidad de reutilizarlos. Como principales desventajas se citan la imposibilidad de entrenar procedimientos completos y sobre todo la baja fidelidad, entendiendo por tal la capacidad del instrumento de 
reproducir lo más exactamente posible la situación que se intenta recrear o simular.

Varios grupos han ido desarrollado distintos programas de entrenamiento con sus respectivos ejercicios o estaciones, que actualmente forman parte de la mayoría de los cursos de laparoscopía básica que se dictan, y que hemos incluido en nuestra experiencia en el Hospital Maciel. En 1992, el Yale Laparoscopic Skills and Suturing Program ${ }^{(10)}$ incluyó tres ejercicios: recorrer una cuerda o cordel, el traslado de porotos a un cubo a través de un pequeño agujero, y el traslado de cilindros con una aguja montada en un instrumento de prehensión. Posteriormente, Scott y colaboradores ${ }^{(11)}$ modificaron estos ejercicios y agregaron otros dos (sutura de espuma y el traslado de 16 letras y números), lo que en su conjunto se conoce como estaciones Southwestern. En 1998, un grupo de la Universidad Mc Gill ${ }^{(12)}$ desarrolló el programa MISTELS (McGill Inanimate System for Training and Evaluation of Laparoscopic Skills). Para ello, un grupo de cirujanos expertos definió siete aspectos que debían entrenarse en laparoscopía básica: visión monocular magnificada, percepción limitada de profundidad, percepción visual-espacial, uso complementario de ambas manos, ligadura de estructura tubular, cortar con precisión mientras la mano no dominante tracciona, y sutura utilizando nudo extra e intracorpóreo. A partir de estos preceptos, desarrollaron cinco ejercicios: traslado de cilindros, corte de un patrón, ligadura con endo-loop, sutura extracorpórea y nudo intracorpóreo. A partir del éxito de este programa y dada la atención que suscitó, en 2004 la SAGES (Society of American Gastrointestinal Endoscopic Surgeons) desarrolló su conocido programa Fundamentals of Laparoscopic Surgery (FLS) ${ }^{(13)}$, y en 2008 el American Board of Surgery (ABS) estableció que todos los aspirantes al título de cirujano debían haber aprobado este curso, entre otros ${ }^{(14)}$.

\section{Otros modelos}

Previo a la introducción de otros modelos de simulación, los únicos disponibles para el entrenamiento en laparoscopía eran los animales vivos y los cadáveres.

Los modelos de entrenamiento en animales vivos tienen como principal ventaja su alta fidelidad, presentando incluso movimientos respiratorios, sangrado, y pueden realizarse procedimientos múltiples y completos. La principal desventaja, además de la logística y el costo que implica el traslado y cuidado de los mismos, así como la imposibilidad de volver a utilizarlos en futuras sesiones de entrenamiento, radica en las limitantes desde el punto de vista ético y legal que en muchos países regula el uso de animales para experimentación, sobre todo vinculadas a la seguridad de los mismos durante todo el proceso. Además, en algunos casos la anatomía no es exactamente igual a la del ser humano, lo que puede limitar aún más su uso.

Los modelos cadavéricos tienen alta fidelidad, y a diferencia de los modelos animales vivos, reproducen exactamente la anatomía. Sin embargo, también presentan importantes desventajas dada su escasa disponibilidad, costos, infraestructura necesaria, pero principalmente por la distinta complacencia que tienen los tejidos.

Por último, citaremos los simuladores de realidad virtual, ampliamente utilizados en el entrenamiento de pilotos de aviación y actualmente disponibles como modelo para simulación en cirugía. Tienen una fidelidad aceptable aunque no tan buena como la de los modelos anteriores, y como desventaja un costo elevado inicial; sin embargo, ofrecen la posibilidad de reutilización con escaso costo adicional en el largo plazo, excepto se deseen incorporar nuevos procedimientos con su correspondiente software. Una de las principales ventajas es que pueden simular escenarios con diversos grados de dificultad, proveer un buen feedback de inmediato, lo que reduce la necesidad de que un mentor/entrenador supervise constantemente los ejercicios, y fundamentalmente la posibilidad de incorporar sistemas de medición que proveen información útil con respecto a la cantidad y precisión de los movimientos efectuados.

\section{Método de entrenamiento}

La simulación entrena fundamentalmente una de las tantas cualidades que hacen al cirujano integral: la habilidad. Otras, como el conocimiento teórico, la experiencia y el juicio clínico en la toma de decisiones, no pueden trasladarse al simulador, por lo que este dificílmente podrá sustituir completamente a la cirugía en escenarios reales.

Una buena estrategia consiste en la deconstrucción $^{(15)}$ de un procedimiento (por ejemplo, una anastomosis intestinal) en sus distintas etapas básicas. De esta forma, el alumno enfoca toda su atención en cada una de dichas etapas en forma ordenada y secuencial, de forma tal que cuando haya dominado la primera pasará a la siguiente, agregándole a la anterior nuevos pasos técnicos y así sucesivamente.

El otro elemento clave es la retroalimentación o feedback. Aquí la simulación ofrece varias ventajas con respecto al aprendizaje en block quirúrgico. En primer lugar, el alumno puede equivocarse sin que esto suponga riesgo alguno. En segundo lugar, la tutorización será guiada y sobre todo estandarizada, con lo que se reduce en gran medida la variabilidad de la información que estos reciben, lo que podemos objetivar a diario en casi todos los servicios de cirugía. Y por último, los alumnos podrán reconocer y corregir sus errores, lo que se conoce como retroalimentación efectiva ${ }^{(16)}$. 
Tabla 1. Escala OSATS para habilidades quirúrgicas generales (22).

\begin{tabular}{|c|c|c|c|c|c|}
\hline Puntuación & Respeto por los tejidos & Tiempo y movimientos & Uso del instrumento & $\begin{array}{l}\text { Tiempo de cirugía y } \\
\text { planificación posterior }\end{array}$ & Conocimientos \\
\hline 1 & $\begin{array}{l}\text { Uso frecuente de fuerza } \\
\text { innecesaria. Daño } \\
\text { causado por uso } \\
\text { inapropiado de los } \\
\text { instrumentos }\end{array}$ & $\begin{array}{l}\text { Muchos movimientos } \\
\text { innecesarios }\end{array}$ & $\begin{array}{l}\text { Repetidamente hace } \\
\text { movimientos vacilantes y } \\
\text { torpes con instrumentos }\end{array}$ & $\begin{array}{l}\text { Frecuentemente detiene } \\
\text { el procedimiento o } \\
\text { requiere discutir los } \\
\text { pasos siguientes }\end{array}$ & $\begin{array}{l}\text { Conocimiento deficiente y } \\
\text { requiere instrucciones en } \\
\text { la mayoría de los pasos } \\
\text { quirúrgicos }\end{array}$ \\
\hline 3 & $\begin{array}{l}\text { Manejo cuidadoso de los } \\
\text { tejidos, pero } \\
\text { ocasionalmente produce } \\
\text { daño inadvertido }\end{array}$ & $\begin{array}{l}\text { Relación tiempo y } \\
\text { movimientos eficiente, } \\
\text { pero realiza algunos } \\
\text { movimientos } \\
\text { innecesarios }\end{array}$ & $\begin{array}{l}\text { Uso competente de los } \\
\text { instrumentos, pero } \\
\text { ocasionalmente se } \\
\text { observa rígido o torpe }\end{array}$ & $\begin{array}{l}\text { Demuestra habilidades } \\
\text { para planear los pasos } \\
\text { siguientes, con } \\
\text { progresión constante del } \\
\text { procedimiento }\end{array}$ & $\begin{array}{l}\text { Conoce todos los } \\
\text { aspectos importantes de } \\
\text { la operación }\end{array}$ \\
\hline 5 & $\begin{array}{l}\text { Maneja los tejidos } \\
\text { apropiadamente con } \\
\text { mínimo daño }\end{array}$ & $\begin{array}{l}\text { Economía de } \\
\text { movimientos y máxima } \\
\text { eficiencia }\end{array}$ & $\begin{array}{l}\text { Movimientos fluidos con } \\
\text { los instrumentos y sin } \\
\text { torpeza }\end{array}$ & $\begin{array}{l}\text { Curso planeado de la } \\
\text { operación en forma } \\
\text { obvia, con flujo sin } \\
\text { esfuerzo }\end{array}$ & $\begin{array}{l}\text { Familiaridad demostrada } \\
\text { con todos los aspectos } \\
\text { de la operación }\end{array}$ \\
\hline
\end{tabular}

Finalmente, es de suponer que la mejor estrategia de entrenamiento, tanto para principiantes como para cirujanos que deben recertificar sus aptitudes, es aquella que se da en forma continua aunque fragmentada ("distributed practice"), en comparación con el entrenamiento intensivo en un período breve de tiempo ("massed practice") ${ }^{(17)}$.

\section{Estrategias de evaluación en simulación}

Un cirujano debe poder desarrollar habilidades técnicas y no técnicas, estas últimas abarcan la toma de decisiones, la capacidad de trabajo en equipo, comunicación y liderazgo, y son tan importantes como las primeras ${ }^{(18)}$. El entrenamiento en simuladores busca reforzar sobre todo las habilidades técnicas, que en cirugía se centran en la destreza manual.

Hay dos tipos de evaluación: sumativa y formativa. La primera determina si un alumno es candidato a participar de un programa de entrenamiento o si ha superado determinada etapa del mismo, es decir que se obtiene únicamente durante la realización de un test o examen. La segunda nos da una idea de la progresión del mismo a lo largo del programa, permitiendo detectar puntos débiles o dificultades y trabajarlos para lograr un aprendizaje eficiente $^{(19)}$.

Un aspecto muy criticado de la metodología de evaluación del desempeño de los cirujanos o residentes, tanto en simuladores como en cirugía real, es que tradicionalmente esta ha sido subjetiva ${ }^{(20)} \mathrm{y}$, por lo tanto, muy difícil de validar, lo que motivó la necesidad de desarrollar herramientas de evaluación objetivas. Una de las más utilizadas es la denominada OSATS (Objective Structured Assesment of Technical Skills) ${ }^{(21)}$ (tabla 1), que abarca aspectos genera- les y específicos del desempeño durante procedimientos quirúrgicos. La misma considera distintos elementos, como respeto por los tejidos, tiempo y movimientos, uso de instrumento, flujo de la cirugía y planificación, y conocimientos, y a su vez cada uno de esos ítems se califica con tres valores $(1,3$ o 5$)$.

La limitante de este tipo de escalas globales es que no evalúan en qué etapa de determinado procedimiento el alumno presenta dificultades, por lo que se han diseñado también escalas específicas para diversos procedimientos quirúrgicos, como la colecistectomía o el by-pass gástrico, quizá con mayor aplicación durante la realización de procedimientos reales en block quirúrgico y no tanto durante el entrenamiento en simuladores, para lo cual la OSATS se considera adecuada.

\section{Desafíos actuales}

Como toda estrategia de enseñanza relativamente novedosa, la simulación enfrenta actualmente algunos desafíos ${ }^{(23)}$.

El primero de ellos es poder contar con recursos humanos adecuadamente capacitados, entre ellos los docentes, que deberán estar acreditados en este tipo de entrenamiento $^{(24)}$. A su vez, la integración definitiva de la simulación a la educación quirúrgica, tanto en los programas de residencia de cirugía como en la educación médica continua, requiere: a) diseñar un programa validado y sustentado por la evidencia científica; b) definir objetivos, herramientas de evaluación y una instancia final de feedback; c) lograr un adecuado balance entre el tiempo dedicado por los alumnos a las actividades asistenciales inherentes a su cargo y a las formativas. Este punto también se 
aplica para los cirujanos ya formados que desean mantener o mejorar su nivel de entrenamiento, lo que requiere asistir a cursos generalmente "intensivos", pero que indefectiblemente restan tiempo a otras actividades laborales de tipo asistencial que suelen ser difíciles de postergar. Se deben considerar algunas limitantes desde el punto de vista logístico, ya que se precisan fondos para costear la adquisición y el mantenimiento del equipamiento necesario, cuyo costo de adquisición puede ser significativo para algunas instituciones, así como del instrumental y otros insumos que deberán reponerse periódicamente. Se debe disponer también de un espacio físico adecuado únicamente destinado a esta actividad. Finalmente, es importante recordar que más allá de estas dificultades, el impacto a largo plazo de la simulación mejoraría el rendimiento del funcionamiento del block quirúrgico, optimizando su utilización en función de las horas de disponibilidad del mismo (dedicando menos tiempo al aprendizaje), lo que significaría una amortización de la inversión. Para demostrarlo, falta realizar aún estudios que analicen la relación costo-eficacia.

\section{Creación de un laboratorio de simulación}

Las actividades de entrenamiento y simulación deben llevarse a cabo en una planta física destinada exclusivamente a las mismas: el laboratorio o centro de simulación. Si bien no es un requisito indispensable, su ubicación próxima o en el mismo centro hospitalario facilitan el acceso de los alumnos, sea mientras desempeñan su actividad regular o incluso durante los días de guardia.

El mismo debe contar con un espacio suficientemente amplio para la ubicación de los "box trainers" junto con sus respectivos monitores, de modo tal que más de un alumno pueda entrenarse simultáneamente en forma independiente.

También dispondrá de un economato en el que se organice y guarden los insumos a utilizar, tanto instrumental quirúrgico como material inerte para los "endotrainers", así como dispositivos especialmente diseñados para descartar material punzo cortante.

El uso de tejido animal ex vivo (por ejemplo, intestino) requiere poder contar, además, con equipamiento adecuado que permita mantenerlo congelado hasta el momento de su utilización.

Todos estos requerimientos deberán adaptarse a las necesidades y sobre todo a las posibilidades. Si bien algunos centros de entrenamiento también llevan a cabo actividades de cirugía experimental, estas conllevan la necesidad de utilizar animales vivos con todo lo que ello implica y que indudablemente no son viables si el centro de simulación se sitúa dentro de una institución médica o centro hospitalario.

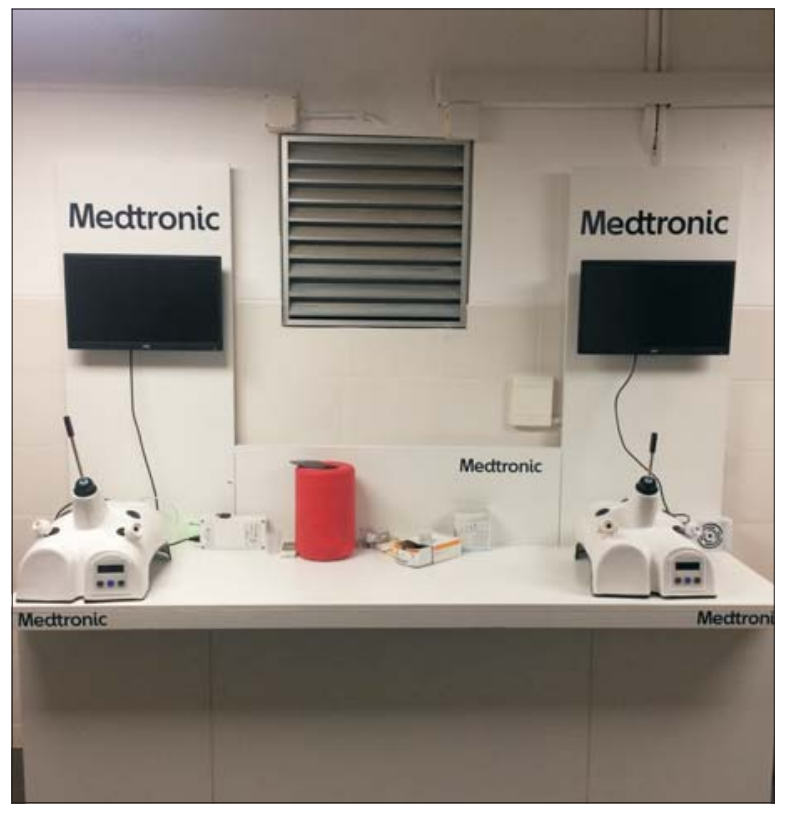

Figura 1. "Endotrainers" en laboratorio de cirugía del Hospital Maciel.

\section{Experiencia inicial en la Clínica Quirúrgica 2 del Hospital Maciel}

Actualmente la realidad de nuestro medio no escapa a la de otros países de la región en cuanto a la formación del cirujano en laparoscopía básica y avanzada, probablemente porque esta depende del lugar donde se cursa la residencia y los recursos con que se cuenta, así como por la motivación personal para incrementar las destrezas laparoscópicas concurriendo a distintos cursos y centros de entrenamiento. Estos lugares, aunque debidamente autorizados y capacitados para ello, carecen formalmente de programas estructurados de entrenamiento en la mayoría de los casos, cuya validez y transferibilidad ha sido ya plenamente demostrada ${ }^{(25)}$.

El Hospital Maciel dispone de una planta física destinada exclusivamente para entrenamiento y simulación a cargo de los Departamentos de Cirugía y de Neurocirugía (Laboratorio de Cirugía y Microcirugía).

El espacio destinado a cirugía general cuenta con dos "endotrainers" cuya cámara puede manipularse mediante un brazo articulado, que transmiten la imagen a sus respectivos monitores de alta definición. Ambos componentes están situados a una altura tal que la posición de los alumnos sea completamente ergonómica (figura 1). También funciona una torre de laparoscopía con ópticas de 0 y 30 grados junto a un "box trainer" que, a diferencia de los otros, no cuenta con fuente de luz propia, por lo que utiliza la fuente de luz de la torre. El instrumental quirúrgico laparoscópico disponible consta 


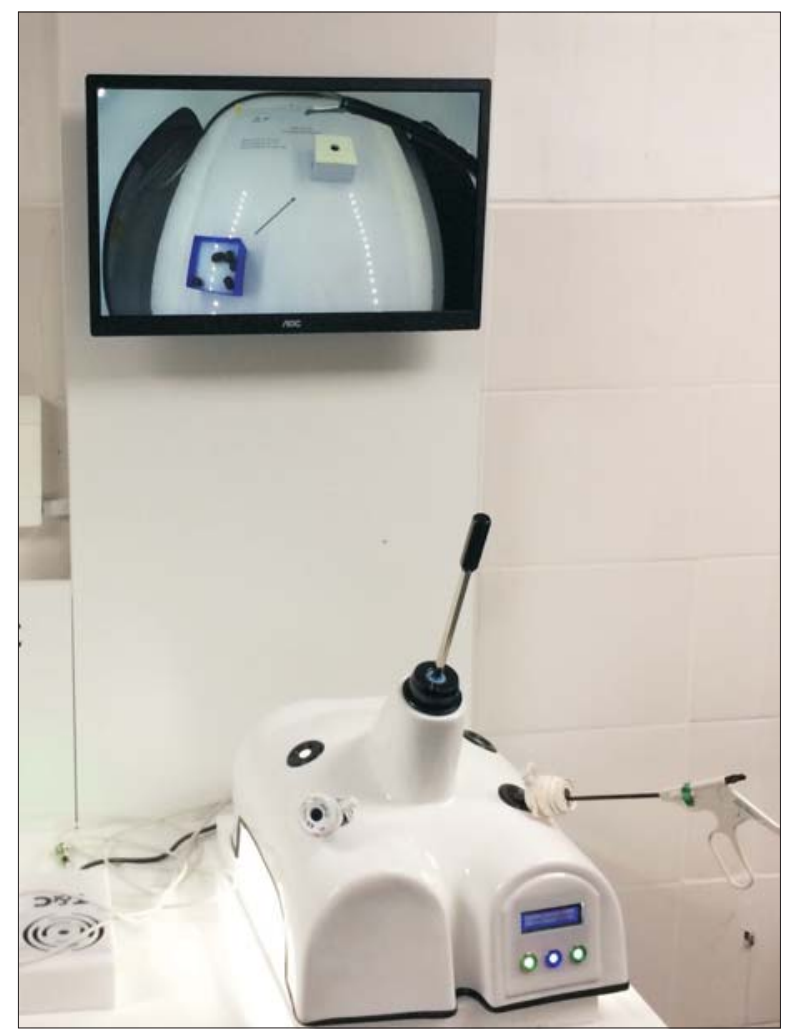

Figura 2. Estaciones "traslado de porotos" (FLS) y "sutura" (Southwestern). Laboratorio de cirugía del Hospital Maciel.

de pinzas Maryland, graspers de prehensión atraumáticas, tijeras, portaagujas y "endo-loop".

Los ejercicios diseñados para llevar a cabo en los "endotrainers" fueron comentados anteriormente (estaciones Southwestern y FLS, figuras 2 y 3 ), tomando para ello como referencia el programa Curso de entrenamiento de habilidades básicas en cirugía laparoscópica, que se realiza en el centro de entrenamiento en habilidades quirúrgicas del Departamento de Cirugía Oriente de la Universidad de Chile. Los objetivos específicos que persigue esta metodología son: conocer los principios básicos de la laparoscopía, manejo de la óptica de 0 y 30 grados, manejo del instrumental laparoscópico básico, orientación espacial en dos dimensiones, manejo de instrumental para mover objetos con precisión y rapidez, desarrollar bimanualidad, uso de "endo-loop", canulación de conducto cístico y realización de nudo simple intracorpóreo.

En setiembre de 2017 se realizó una jornada inicial a modo de experiencia piloto en la que todos los residentes de cirugía general del Hospital Maciel pudieron conocer y familiarizarse con los "box-trainers" y los diferentes ejercicios o tareas. Posteriormente, aquellos que se encontraban cursando el primer y segundo año de la

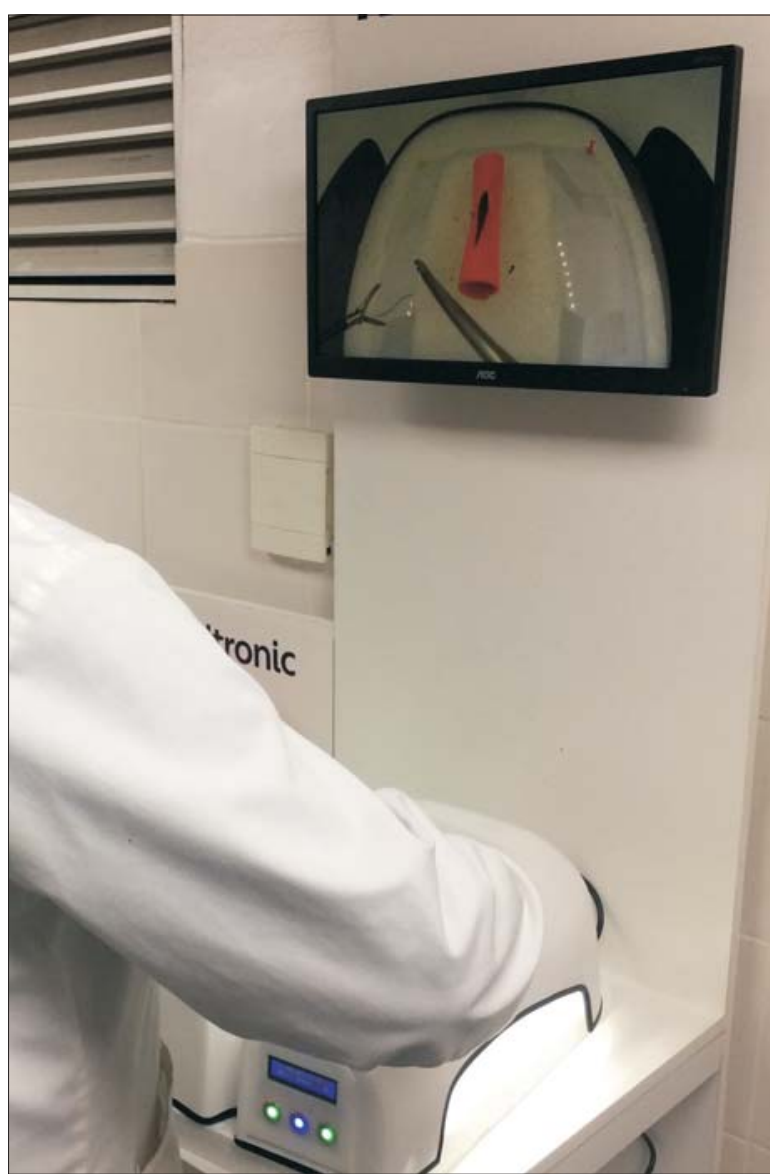

Figura 3. Estaciones "traslado de porotos" (FLS) y "sutura" (Southwestern). Laboratorio de cirugía del Hospital Maciel.

residencia comenzaron un programa de entrenamiento básico, en sesiones de entre 60-90 minutos de duración, dos o tres veces a la semana, hasta lograr los objetivos planteados, lo que demandó tres meses en total. A partir de este año en nuestro servicio de cirugía se estableció, como requisito para poder comenzar a realizar procedimientos quirúrgicos laparoscópicos de baja complejidad, que los residentes de primer año completasen el mismo programa, con igual carga horaria y frecuencia semanal. La actividad de simulación se llevó a cabo en un tiempo total de diez semanas (abril a junio de 2018), lo que equivale a unas 20 a 30 horas de entrenamiento total promedio, durante la cual los alumnos fueron supervisados por un asistente de Clínica Quirúrgica previamente capacitado y designado para dicha tarea. La aprobación del mismo se logró una vez realizados todos los ejercicios siguiendo estrictamente la técnica descrita, pero sobre todo logrando tiempos de suficiencia previamente establecidos en dos oportunidades o tests para cada uno. Se le asignó a cada alumno una planilla de registro del tiempo cronometrado para todos los ejercicios 


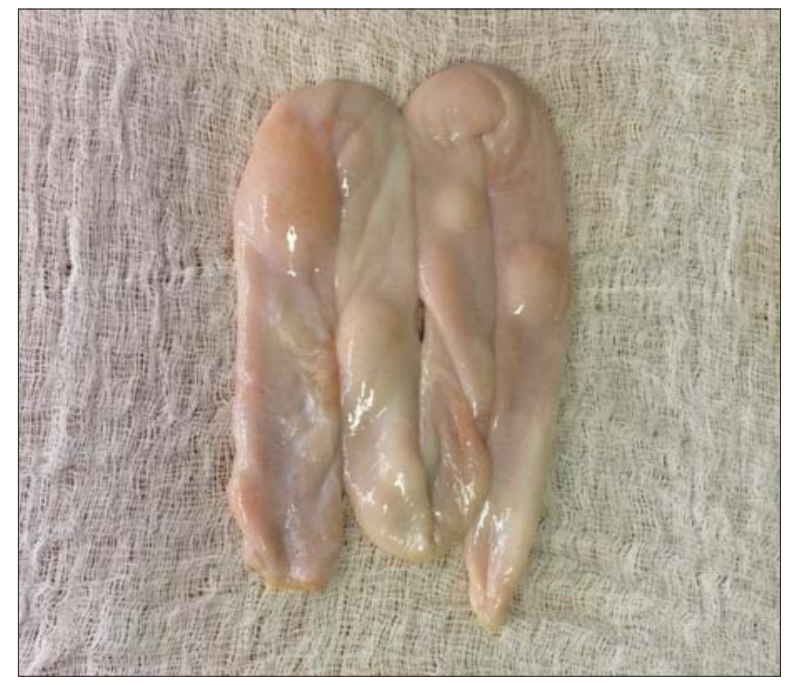

Figura 4. Tejido animal ex vivo para modelo de entrenamiento avanzado. Tomado del Centro de entrenamiento en habilidades quirúrgicas, Facultad de Medicina de la Universidad de Chile.

(de cada ensayo y de las pruebas finales), de modo que los mismos objetivaran su progresión a medida que transcurría el curso, con disminución paulatina de los tiempos hasta lograr la suficiencia.

Al mismo tiempo se llevó a cabo un curso de laparoscopía para todos los residentes de cirugía del hospital en mayo de 2018, como complemento del programa de entrenamiento para aquellos que ya lo habían completado y los que los estaban realizando. Este curso, de seis horas de duración, que contó además con una actividad práctica en los simuladores, tuvo como objetivo principal brindar conocimientos teóricos acerca de la laparoscopía y sus aspectos más relevantes (incluidos los fundamentos de la simulación), como son: vías de acceso para la creación del neumoperitoneo y los cambios fisiopatológicos producto de ello, complicaciones durante el acceso a la cavidad peritoneal e instrumental laparoscópico (básico, selladores, bisturí ultrasónico, endograpadoras).

De esta forma, hemos comenzado a transitar por un camino relativamente novedoso en nuestro medio a nivel institucional, realizando cursos teóricos junto con actividades de simulación en laparoscopía. Estos han sido dirigidos a los residentes que comienzan su formación quirúrgica, en una modalidad de programa de entrenamiento básico estructurado y validado, cuyo resultado podemos decir que ha sido plenamente satisfactorio. Sin embargo, la principal limitante o dificultad que detectamos guarda relación con la coordinación de estas actividades con respecto a la tarea asistencial-académica (y el balance entre ellas), ya que tanto alumnos como docentes participan regularmente de ambas. No obstante, constituye un desafío y un estímulo para seguir por esta línea de trabajo y a partir de ello es que nuestro próximo objetivo será realizar un programa de entrenamiento de laparoscopía avanzada, dirigido no solo a todos aquellos cursantes que hayan completado y aprobado el módulo o programa básico, sino también a los cirujanos titulados que deseen adquirir, mejorar o mantener un entrenamiento para llevar a cabo procedimientos de laparoscopía avanzada. Este programa ya fue concebido y diseñado ${ }^{(7)}$, pero, a diferencia del anterior, requiere determinados insumos básicos (tejidos ex vivo como el intestino porcino, figura 4), para los cuales debe adecuarse la infraestructura necesaria, ya que los mismos deben mantenerse conservados congelados y prepararse previo a cada sesión de entrenamiento, sin posibilidad de reutilización.

Creemos que con el apoyo necesario nuestro centro de entrenamiento y simulación puede llegar a ser un punto de referencia en esta área. Para ello es clave estandarizar o sistematizar los programas básico y avanzado, y también formar y acreditar adecuadamente los recursos humanos docentes a cargo de los mismos.

\section{Summary}

Simulation arises as a fundamental tool within the paradigm shift in the current teaching of surgery. It is based on modern educational theories, patient safety and, in the case of laparoscopy the inherent difficulties of the technique. The main objective is to transfer the training in motor skills outside the operating room, to the simulation laboratory. For this purpose, there are several simulation models, like box trainers -among others- , which despite its low fidelity, adds several advantages in relation to its cost, availability and reuse. This study presents a descriptive review of the subject, including the different models and training methods, evaluation strategies, as well as our initial experience in the Surgical Clinic 2 of the Maciel Hospital

\section{Resumo}

A simulação surge como uma ferramenta fundamental no cambio de paradigma do ensino da cirurgia no qual trabalhamos atualmente, baseado em teorias educativas modernas, na segurança do paciente e, no caso da laparoscopia, nas dificuldades próprias da técnica. O objetivo fundamental é transferir o treinamento das habilidades motoras do centro cirúrgico ao laboratório de simulação. Existem diversos modelos de simulação, entre os quais destacamos os box trainers, que apesar de sua baixa fidelidade, apresentam varias vantagens em relação a custos, disponibilidade e reutilização. Neste artigo faze- 
mos uma revisão descritiva do tema, incluindo os diferentes modelos e métodos de treinamento, estratégias de avaliação, e também da experiência inicial na Clínica Quirúrgica 2 do Hospital Maciel.

\section{Bibliografía}

1. Carter BN. The fruition of Halsted's concept of surgical training. Surgery 1952; 32:518-27.

2. Fitts PM, Posner MI. Human performance. Belmont, CA: Brooks/Cole, 1967.

3. Kohn LT, Corrigan JM, Donaldson MS, eds. To err is human: building a safer heath system. Washington, DC: National Academy Press; 1999.

4. Peters JH, Fried GM, Swanstrom LL, Soper NJ, Sillin LF, Schirmer B, et al. Development and validation of a comprehensive program of education and assessment of the basic fundamentals of laparoscopic surgery. Surgery 2004; 135:21-7.

5. Wanzel KR, Hamstra SJ, Caminiti MF, Anastakis DJ, Grober ED, Reznick RK. Visual-spatial ability correlates with effciency of hand motion and successful surgical performance. Surgery 2003; 134:750-7.

6. Reznick R, MacRae H. Teaching surgical skills - changes in the wind. N Engl J Med 2006; 355:2664-9

7. Varas J, Mejía R, Riquelme A, Maluenda F, Buckel E, Salinas J, et al. Significant transfer of surgical skills obtained with an advanced laparoscopic training program to a laparoscopic jejunojejunostomy in a live porcine model: feasibility of learning advanced laparoscopy in a general surgery residency. Surg Endosc 2012; 26:3486-94.

8. McGaghie WC, Issenberg SB. Simulation in professional competence assessment: basic considerations. In: Tekian A, McGuire $\mathrm{CH}$, McGaghie WC, eds. Innovative simulations for assessing professional competence. Chicago: University of Illinois at Chicago, Department of Medical Education, 1999:7-22.

9. Bholat OS, Haluck RS, Murray WB, Gorman PJ, Krummel TM. Tactile feedback is present during minimally invasive surgery. J Am Coll Surg 1999; 189:349-55.

10. Rosser JC, Davis BR, Qureshi HN. Intracorporeal suturing: the top gun experience. In: Fischer JE, Bland KI, eds. Mastery of surgery. $5^{\text {th }}$ ed. Philadelphia (PA): Lippincott, Wi1liams \& Wilkins; 2007:2592 v.2.

11. Scott DJ, Bergen PC, Rege RV, Laycock R, Tesfay ST, Valentine RJ, et al. Laparoscopic training on bench models: better and more cost effective than operating room experience? J Am Coll Surg 2000; 191(3):272-83.

12. Derossis AM, Fried GM, Swanstrom LL, Sigman HH, Barkun JS, Meakins JL. Development of a model for trai- ning and evaluation of laparoscopic skills. Am J Surg 1998; 175(6):482-7.

13. Peters JH, Fried GM, Abrahamowicz M, Soper NJ, SiIlins LF, Schirmer B, et al. Development and validation of a comprehensive program of education and assessment of the basic fundamentals of laparoscopic surgery. Surgery 2004; 135(1):21-7.

14. Soper NJ, Fried GM. The fundamentals of laparoscopic surgery: its time has come. Bull Am Coll Surg 2008; 93(9):30-2.

15. Hirsch ED. The core knowledge curriculum —what's behind its success? Educ Leadership 1993; 50:23-5.

16. Kolozsvari N, Feldman L, Vassiliou M, Demyttenaere S, Hoover M. Sim one, do one, teach one: considerations in designing training curricula for surgical simulation. J Surg Educ 2011; 68:421-7.

17. Mackay S, Morgan P, Datta V, Chang A, Darzi A. Practice distribution in procedural skills training: a randomized controlled trial. Surg Endosc 2002; 16:957-61.

18. Baldwin PJ, Paisley AM, Brown SP. Consultant surgeons' opinion of the skills required of basic surgical trainees. Br J Surg 1999; 86:1078-82.

19. Aggarwal R, Grantcharov TP, Darzi A. Framework for systematic training and assessment of technical skills. J Am Coll Surg 2007; 204:697-705.

20. Wanzel KR, Ward M, Reznick RK. Teaching the surgical craft: from selection to certification. Curr Probl Surg 2002; 39:573-659.

21. Martin JA, Regehr G, Reznick R, MacRae H, Murnaghan J, Hutchison C, et al. Objective structured assessment of technical skill (OSATS) for surgical residents. Br J Surg 1997; 84:273-8.

22. Corvetto MA, Bravo MP, Montaña RA, Altermatt FR, Delfino AE. Inserción de la simulación clínica en el currículum de Anestesiología en un hospital universitario. Evaluación de la aceptabilidad de los participantes. Rev Esp Anestesiol Reanim 2013; 60:320-6.

23. Stefanidis D, Sevdalis N, Paige J, Zevin B, Aggarwal R, Grantcharov T, et al. Simulation in surgery: what's needed next? Ann Surg 2015; 261(5):846-53.

24. Hull L, Arora S, Symons NR, Jalil R, Darzi, A, Vincent C, et al. Training faculty in nontechnical skill assessment: national guidelines on program requirements. Ann Surg 2013; 258:370-5.

25. Sroka G, Feldman LS, Vassiliou MC, Kaneva PA, Fayez R, Fried GM. Fundamentals of laparoscopic surgery simulator training to proficiency improves laparoscopic performance in the operating room-a randomized controlled trial. Am J Surg 2010; 119:115-20. 